\title{
Total antioxidant status (TAS) in childhood cancer survivors
}

\section{Maryna Krawczuk-Rybak ${ }^{1}$, Anna Panasiuk ${ }^{1}$, Małgorzata Czygier ${ }^{2}$, Katarzyna Muszynska-Roslan ${ }^{1}$, Jolanta Wysocka ${ }^{3}$, Maciej Szmitkowski ${ }^{2}$}

\author{
${ }^{1}$ Department of Pediatric Oncology and Hematology, Medical University of Bialystok, Poland \\ ${ }^{2}$ Department of Biochemical Diagnostics, Medical University of Bialystok, Poland \\ ${ }^{3}$ Department of Pediatric Laboratory Diagnostics, Medical University of Bialystok, Poland
}

\begin{abstract}
Total antioxidant status (TAS), and the influence of treatment and correlation between TAS and parameters involved in metabolic syndrome (MS) in pediatric cancer survivors were evaluated. One hundred children and adolescents were studied. Twenty-five survivors received radiotherapy, 12 were obese or overweight. Additionally, we analyzed TAS in eight children with acute lymphoblastic leukemia (ALL) at diagnosis and during treatment after remission induction. The control group consisted of 22 healthy children. Serum concentrations of TAS, glucose, cholesterol, HDL-cholesterol, triglycerides, fibrinogen and insulin were measured. In cancer survivors, independently of diagnosis and kind of treatment (radiotherapy anthracyclines administration), the mean serum TAS did not differ significantly from the control group. No correlations were observed with age at the time of diagnosis or interval after the end of treatment. TAS values did not correlate with traits of the metabolic syndrome. In a group of eight patients with ALL at diagnosis and after induction of remission, TAS values were lower than in the control and cancer survivor groups. Antioxidant status was not found to be deteriorated in children after anticancer treatment, irrespective of diagnosis or kind of treatment, which might indicate sufficient antioxidant prevention. However, the possibility of the development of MS and cardiovascular disease in adulthood indicates the need for future studies. (Folia Histochemica et Cytobiologica 2012, Vol. 50, No. 3, 468-472)
\end{abstract}

Key words: cancer survivors, children, antioxidants, metabolic syndrome

\section{Introduction}

Oxidative stress, measured as reactive oxygen species (ROS), plays an important role in cancerogenesis due to DNA damage and its modification [1]. Anticancer treatment, radiotherapy and chemotherapy (i.e. cyclophosphamide, anthracyclines) induce ROS production. This leads to the death of neoplastic cells, but may also influence damage to healthy cells and tissues [2-4].

Correspondence address: M. Krawczuk-Rybak, Department of Pediatric Oncology and Hematology, Medical University of Bialystok,

Waszyngtona Str. 17, 15-274 Bialystok, Poland; e-mail:rybak@umwb.edu.pl
Antioxidant defense is composed of enzymatic (superoxide dismutase - SOD, catalase - CAT, glutathione peroxidase - GTX), and non-enzymatic (vitamin A, vitamin E, vitamin C, thiol antioxidants, albumin, bilirubin, uric acid) antioxidants. Total antioxidant status (TAS) consists of all antioxidants present in body fluids. At the time of cancer diagnosis, and during treatment, the level of antioxidants is decreased, which can lead to impaired function of organs such as the heart muscle, lungs or central nervous system [2, 5-7].

The increasing number of childhood cancer survivors has prompted studies into the late effects of anticancer treatment. It is well known that anticancer therapy damages different organs, not only during treatment, but also leads to their deteriorated function many years later. Late cardiotoxicity, lung dam- 
age, and metabolic syndrome are observed in childhood cancer survivors. In the development of the sequelae, ROS and the antioxidant status play an important role $[3,4,8,9]$.

Until now, there has been no research into the relationship between TAS and metabolic syndrome (MS) in children previously treated for cancer. In the present study, we evaluated total antioxidant status in children after anticancer therapy, as well as the influence of different methods of previously received treatment, age at the beginning of the therapy, and correlation with the different traits involved in MS.

\section{Material and methods}

Patients. One hundred children and adolescents from the Outpatient Clinic of the Department of Pediatric Oncology and Hematology, Medical University of Bialystok, aged from 4.22 to 22.6 years, treated previously for different cancers, were studied. The patients were scheduled for examination after at least two years off therapy, as a follow-up study of cancer survivors. All of them were in the first remissiona mean of $5.3 \pm 2.99$ years after the end of treatment. Twenty-five survivors received radiotherapy. In this group, 14 patients had central nervous system irradiation due to leukemias (dose of 12 or $18 \mathrm{~Gy}$ ) and 11 were irradiated for solid tissue sarcomas or Hodgkin's lymphoma. All children received normal diet and were in good general condition at the time of the study. None of the survivors presented overt or subclinical symptoms of cardiomyopathy, pulmonary dysfunction, hypertension or hormonal dysfunctions. Twelve patients were obese or overweight (body mass index, BMI $>85^{\text {th }}$ percentile).

Additionally, we analyzed TAS at diagnosis and after induction of treatment in eight children diagnosed with ALL in 2008. All of them received the same protocol for ALL treatment and were in general good condition at the measurements (infection and acute organ damage to the lungs, kidneys and heart were excluded). The control group for TAS evaluation consisted of 22 healthy children with a mean age of $12.6 \pm 4.79$ years. Table 1 presents the patients' characteristics.

Methods. Blood samples were collected after 12 hours of fasting, centrifuged, and then immediately frozen and stored at $-80^{\circ} \mathrm{C}$. The serum concentration of TAS was assayed using the enzymatic method with peroxidase by commercially available RANDOX TAS kits (Randox, Ardmore, UK) according to the manufacturer's instructions. In this method, ABTS ${ }^{\circledR}$ (2,2 Azino-di-[3-ethylbenzthiazoline sulphonate) is incubated with a peroxidase metmyoglobin and $\mathrm{H}_{2} \mathrm{O}_{2}$ to produce the radical cation $\mathrm{ABTS} \AA^{*}+$. This product has a relatively stable blue-green color, which was measured at $600 \mathrm{~nm}$. Antioxidants in the added serum sample cause sup-
Table 1. Patients' profile in the study group

\begin{tabular}{|l|c|}
\hline Characteristics & \\
\hline Gender & \\
Male (n, \%) & $58(58 \%)$ \\
Female (n, \%) & $42(42 \%)$ \\
\hline Age at study & 12.74 \\
(years; median [1 ${ }^{\text {st }}$ and $3^{\text {rd }}$ quartile]) & $(8.68 ; 16.55)$ \\
\hline Age at diagnosis (years, mean \pm SD) & $7.0 \pm 4.94$ \\
\hline Age at cessation of treatment & $8.7 \pm 4.89$ \\
(years, mean \pm SD) & \\
\hline Years since cessation of treatment & $5.3 \pm 2.99$ \\
(mean \pm SD) & \\
\hline Diagnosis & \\
Acute lymphoblastic leukemia (n, \%) & $49(49 \%)$ \\
Acute non-lymphoblastic leukemia (n, \%) & $3(3 \%)$ \\
Non-Hodgkin's Lymphoma (n, \%) & $11(11 \%)$ \\
Hodgkin's Disease (n, \%) & $4(4 \%)$ \\
Solid tumors (n, \%) & $33(33 \%)$ \\
& \\
\hline Dose of radiotherapy (n, \%) & $10(40 \%)$ \\
1,200 Gy (central nervous system) & $4(16 \%)$ \\
1,800 Gy (central nervous system) & $11(44 \%)$ \\
> 1,800 Gy (supra- and infradiaphragmatic & \\
irradiation) & \\
\hline
\end{tabular}

pression of this color production to a degree which is proportional to their concentration.

Biochemical tests with automated direct methods (with commercial kits) were used to analyze serum glucose (enzymatic method with hexokinase, Roche) cholesterol (CHOL) (enzymatic method with cholesterol oxidase, Roche), HDL-cholesterol (HDL) (direct measurement with polyethylene glycolmodified enzymes, PEG, Roche), triglycerides (TG) (with phosphoglycerol oxidase method, Roche), fibrinogen (photo-optical method, Multifibren U, DADE Behring Diagnostics), insulin (chemiluminescence immunoassay, Insulin, Immulite Siemens).

Measurements of weight, height, and blood pressure were obtained using conventional techniques. Body mass index (BMI) was calculated as weight in kilograms divided by height in square meters. Abdominal obesity was calculated from waist-to-hip ratio.

The study was approved by the Ethical Committee of the Medical University of Bialystok. Written informed consent was obtained from parents and adolescents ( $>16$ years).

Statistical analysis was performed using GraphPad Prism 4.0 version. Data was expressed as means \pm standard deviations, or when appropriate (depending on skewness) as median and quartiles. Kruskal-Wallis test was used to examine differences in continuous variables between patients in different diagnosis and treatment groups. For comparison of nonparametric data sets, Mann-Whitney $U$ test was used. Spearman's test was used to assess correlation between different treatment and time parameters with TAS values. A p value $<0.05$ was considered significant. 
Table 2. Correlation between treatment features and TAS values

\begin{tabular}{|l|c|c|}
\hline Feature & $\begin{array}{c}\text { TAS value } \\
\text { correlation }\end{array}$ & p \\
\hline Age at diagnosis & 0.03 & 0.81 \\
\hline Age at time of study & 0.02 & 0.92 \\
\hline $\begin{array}{l}\text { Time elapsed since cessation } \\
\text { of treatment }\end{array}$ & 0.10 & 0.40 \\
\hline Dose of radiotherapy & -0.01 & 0.95 \\
\hline
\end{tabular}

\section{Results}

In the analyzed group of cancer survivors, the mean serum TAS did not differ significantly from the values measured in the control group. We found no differences in TAS after group subdivision according to diagnosis. No correlations were observed with age at the time of diagnosis, at the time of study, or in the time interval between the end of treatment and this study. There was no effect of previous treatment, namely use of anthracycline (TAS value in group of patients with anthracyclines $1.26 \pm 0.214$ vs. patients without anthracyclines $1.26 \pm 0.180 ; \mathrm{p}=0.91)$ or radiotherapy administration (Table 2 ).

Twelve survivors presented overweight or obesity. We found no differences in TAS values in children with normal weight compared to overweight and obese patients. TAS values did not correlate with parameters describing the metabolic syndrome: BMI, total cholesterol, HDL-CHOL, fibrinogen, fasting and postprandial glucose and insulin, systolic and diastolic blood pressure. We found a positive correlation between TAS and triglyceride values (Table 3 ).

We found no differences in TAS values between groups of patients with two or more elements of MS compared to the remaining patients $(\mathrm{p}=0.38)$.

Additionally, we examined TAS in a small group of eight ALL patients: at diagnosis (before treatment) and during treatment (after induction chemotherapy), TAS values were lower than in the control group and in cancer survivors (Kruskal-Wallis test $\mathrm{p}=0.018$ ) (Figure 1).

\section{Discussion}

In our study, normal TAS values were found in all cancer survivors at a mean 5.3 years after the end of treatment, irrespective of the type of cancer and the treatment received. However, in a small group consisting of eight children before and during treatment, TAS levels were observed to decrease.
Table 3. Correlation of TAS values and different laboratory and clinical findings

\begin{tabular}{|l|c|c|}
\hline Feature & $\begin{array}{c}\text { TAS value } \\
\text { correlation }\end{array}$ & p \\
\hline BMI & 0.15 & 0.25 \\
\hline Total cholesterol & -0.08 & 0.41 \\
\hline HDL-cholesterol & -0.15 & 0.14 \\
\hline Triglycerides & 0.24 & 0.02 \\
\hline Fibrinogen & -0.01 & 0.89 \\
\hline Fasting glucose & 0.18 & 0.08 \\
\hline Postprandial glucose & 0.03 & 0.81 \\
\hline Fasting insulin & 0.22 & 0.05 \\
\hline Postprandial insulin & 0.14 & 0.27 \\
\hline
\end{tabular}

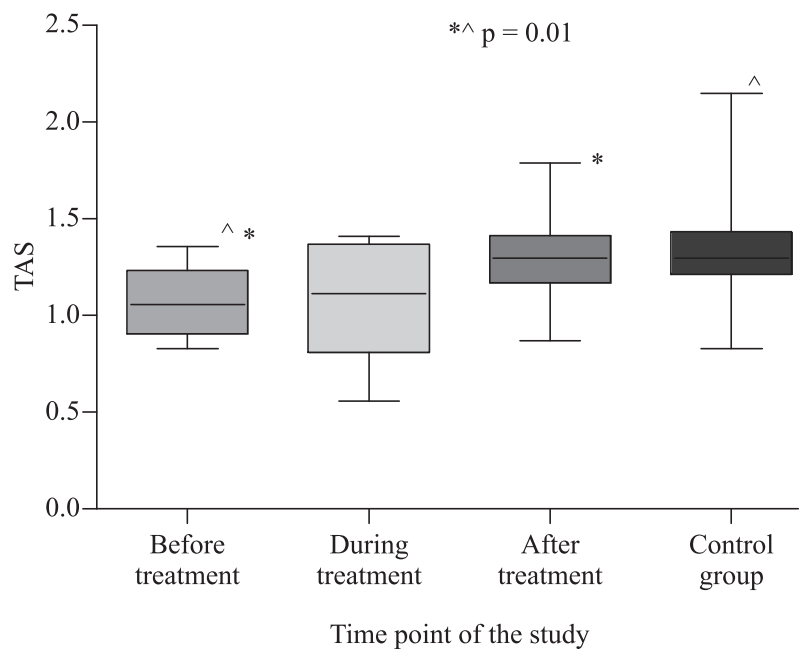

Figure 1. TAS values in children before, during and after the treatment for pediatric cancer, and in control group ( $p$ value for comparison of four studied groups of patients $\mathrm{p}=0.018$; ^ comparison of patients before therapy with control group; *comparison of patients before and after treatment)

The role of ROS in neoplastic transformation and in the development of different cancers has been confirmed by numerous studies in adults and children. Neoplastic cells produce higher amounts of ROS than normal cells. The antioxidant system is altered; the activity of SOD, CAT is reduced and serum vitamin $\mathrm{E}$ is lower, which leads to an imbalance between the oxidative and antioxidative status [1]. Anticancer treatment also influences redox status, i.e. some cytostatic agents act by inducing oxidative stress, neoplastic cell injury and death $[2,4,5,11]$. Some authors have suggested that a high level of the apoptotic index can be a marker of good clinical response to treatment [2]. In childhood leukemia, repeated chemotherapy protocols contribute to prolonged oxida- 
tive stress, which is associated with the intensity of treatment $[5,10]$. The decreased TAS values observed in our study before and after intensive treatment in ALL patients indicate a lack of balance between elevated ROS generation and antioxidant capacity, which is inefficient. This situation can lead to deteriorated function of different organs.

Elevated oxidative stress can cause adverse side effects of treatment, such as cardiotoxicity after anthracyclines or hemorrhagic cystitis after cyclophosphamide $[12,13]$. Anthracyclines generate ROS and cause myocyte injury. Cardiac myocytes are highly susceptible to oxidative damage due to their intensive oxidative metabolism and relatively poor antioxidant defense [4]. Additionally, mediastinal or lung radiotherapy influences the generation of ROS, leading to inflammation, endothelial injury to the heart muscle, and to the future development of atherosclerosis and postradiation fibrosis [2]. Development of postradiation chronic oxidative stress leads to an imbalance between ROS and the antioxidant defense, which results in injury to different tissues and organs $[3,9,14]$.

The systematic progress in anticancer treatment in children over the last 40 years has contributed to an increase in the population of pediatric cancer survivors. Their health status and quality of life are an important problem. Second malignancies, cardiovascular and pulmonary sequelae are the commonest factors influencing late morbidity and mortality of childhood cancer survivors $[8,15,16]$. Oxidative stress and antioxidative status are considered important elements in the development of these late effects [3,4]. Some studies have indicated a favorable influence of diet with antioxidant supplementation on the reduction in the toxic effect of ROS-generating chemotherapies, as well as on a reduced risk of cancer development or recurrence [5, 17-19]. There have been few studies concerning oxidative stress and/or the antioxidant status in children during and after anticancer treatment. All of them were conducted directly after treatment $[7,10]$.

In our study group, all cancer survivors were in the first remission, and none of them presented the clinical signs of cardiovascular or pulmonary dysfunction. We did not find decreased TAS values in any of our survivors, even in those examined a short time (seven months) after the end of treatment.

Our results indicate that previous radiotherapy and therapy with anthracyclines had no lasting effect on the antioxidant status, and even a short time after the treatment was sufficient for antioxidant status reconstruction. Similar findings were presented by Battisti et al., who observed normal CAT and SOD activity in children and young adults after cessation of leukemia treatment [10].
In our cancer survivors, we assessed a possible correlation between TAS and the traits of metabolic syndrome (MS). Truncal obesity, hypertension, hypertriglyceridemia, lowered HDL-cholesterol level, and hyperglycemia are the main components of MS. In children with full or partial MS, reduced TAS, decreased levels of plasma antioxidant vitamins and increased oxidative stress with higher lipid peroxidation are observed. These metabolic alterations lead to endothelial dysfunction and precocious development of atherosclerosis in adulthood [20-24]. In a Dutch analysis, the MS criteria were observed in $13 \%$ of long-term adult survivors of childhood cancers, especially after the treatment for brain tumors or acute leukemias with cranial irradiation [25]. In our study, none of the analyzed survivors presented all the elements characteristic of MS, yet some of them demonstrated obesity and hypertriglyceridemia. We found a positive correlation between TAS and TG levels, but no correlation with other MS parameters. Twelve survivors were overweight, but TAS was not altered in this subgroup. None of the analyzed patients were hypertensive or demonstrated glucose metabolism alterations. This indicates that overweight survivors without other signs of MS present normal antioxidant capacity. Similar observations were made by Molnar et al., who noticed reduced TAS values only in obese children with multimetabolic syndrome, but in the obese patients without MS the values of TAS were normal [22]. The mechanism of MS development in childhood cancer survivors depends on the tumor location (i.e. hypothalamus) and adverse effect of treatment, i.e. central nervous system radiotherapy and/or subsequent growth hormone deficiency, thyroid dysfunction, hypogonadism, steroid therapy [26-28]. In our study group, children irradiated for central nervous system received a dose of 12 or 18 Gy. These doses influence growth hormone production and lead to the development of obesity only to a small degree [29]. At the time of study, all patients were euthyreotic, without signs of hypogonadism.

In conclusion, the antioxidant status in children after anticancer treatment was not found to be deteriorated, irrespective of diagnosis or method of treatment, which might indicate sufficient antioxidative prevention. However, the possibility of MS development and cardiovascular disease in adulthood suggests that future studies in this area are necessary.

\section{References}

1. Klaunig JE, Kamendulis LM, Hocevar BA. Oxidative stress and oxidative damage in carcinogenesis. Toxicol Pathol. 2010;38:96-109.

2. Al-Tonbary $\mathrm{Y}, \mathrm{Al}$-Hasan SA, Zaki M et al. Impact of antioxidant status and apoptosis on the induction phase of chemotherapy in childhood acute lymphoblastic leukemia. $\mathrm{He}$ matology. 2011;1:1-19. 
3. Yarnold J, Brotons MC. Pathogenetic mechanisms in radiation fibrosis. Radiother Oncol. 2010:97:149-161.

4. Trachtenberg BH, Landy DC, Franco VI et al. Anthracyclineassociated cardiotoxicity in survivors of childhood cancer. Pediatr Cardiol. 2011;32:342-353.

5. Kennedy DD, Ladas EJ, Rheingold SR, Blumberg J, Kelly KM. Antioxidant status decreases in children with acute lymphoblastic leukemia during the first six months of chemotherapy treatment. Pediatr Blood Cancer. 2005;44:378-385.

6. Stenzel SL, Krull KR, Hockenberry M et al. Oxidative stress and neurobehavioral problems in pediatric acute lymphoblastic leukemia patients undergoing chemotherapy. J Pediatr Hematol Oncol. 2010;32:113-118.

7. Caron JE, Krull KR, Hockenberry $\mathrm{M}$ et al. Oxidative stress and executive function in children receiving chemotherapy for acute lymphoblastic leukemia. Pediatr Blood Cancer. 2009;53:551-556.

8. Bhatia S, Constine LS. Late morbidity after successful treatment of children with cancer. Cancer J. 2009;15:174-180.

9. Shankar SM, Marina N, Hudson MM et al. Monitoring for cardiovascular disease in survivors of childhood cancer: report from the cardiovascular disease task force of the children's oncology group. Pediatrics. 2008;21:e387-e396.

10. Battisti V, Maders LD, Bagatini MD et al. Measurement of oxidative stress and antioxidant status in acute lymphoblastic leukemia patients. Clin Biochem. 2008:41:511-518.

11. Kasapowić J, Pejić S, Stoilijković V et al. Antioxidant status and lipid peroxidation in the blood of breast cancer patients of different ages after chemotherapy with 5-fluorouracil, doxorubicin and cyclophosphamide. Clin Biochem. 2010;43:16-17.

12. Mazor D, Abucoider A, Meyerstein N, Kapelushnik J. Antioxidant status in pediatric acute lymphocytic leukemia (ALL) and solid tumors: the impact of oxidative stress. Pediatr Blood Cancer. 2008;51:613-615.

13. Šiműnek T, Štërba M, Popelowá O et al. Anthracycline-induced cardiotoxicity: Overview of studies examining the roles of oxidative stress and free cellular iron. Pharmacol Reports. 2009;61:154-171.

14. Zhao W, Diz DI, Robbins ME. Oxidative damage pathways in relation to normal tissue injury. Br J Radiol. 2007;80:S23$-\mathrm{S} 31$.

15. Bhatia S, Constine LS. Late morbidity after successful treatment of children with cancer. Cancer J. 2009;15:174-180.

16. Fryer C. Late effects in childhood cancer survivors: a review with a framing effect bias? Pediatr Blood Cancer. 2011;10:1-4.

17. Block KI, Koch AC, Mead MN et al. Impact of antioxidant supplementation on chemotherapeutic toxicity: A systematic review of the evidence from randomized controlled trials. Int J Cancer. 2008;123:1227-1239.

18. Yeon JY, Suh YI, Kim SW et al. Evaluation of dietary factors in relations to the biomarkers of oxidative stress and inflammation in breast cancer risk. Nutrition. 2011;9:912-918.

19. Sakhi AK, Břhn SK, Smeland S et al. Postradiotherapy plasma lutein, á-carotene, and â-carotene are positively associated with survival in patients with head and neck squamous cell carcinoma. Nutr Cancer. 2010;62:322-328.

20. Dimitrijevic-Sreckovic V, Colak E, Djordjevic P et al. Prothrombogenic factors and reduced antioxidative defense in children and adolescents with pre-metabolic and metabolic syndrome. Clin Chem Lab Med. 2007;45:1140-1144.

21. Ondrejovićowá I, Muchowá J, Misianová C, Nagyová Z, Duraćková Z. Hypercholesterolemia, oxidative stress and gender dependence in children. Prague Med Rep. 2010;111:300-312.

22. Molnár D, Desci T, Koletzko B. Reduced antioxidant status in obese children with multimetabolic syndrome. Int J Obes Relat Metab Disord. 2004:28:1197-1202.

23. Gianinini C, Giorgis T, Scarinci A et al. Increased carotid intima-media thickness in pre-pubertal children with constitutional leanness and severe obesity: the speculative role of insulin sensitivity, oxidant status, and chronic inflammation. Eur J Endocrinol. 2009;161:73-80.

24. Karamouzis I, Pervanidou P, Berardelli R et al. Enhanced oxidative stress and platelet activation combined with reduced antioxidant capacity in obese prepubertal and adolescent girls with full or partial metabolic syndrome. Horm Metab Res. 2011:43:607-613.

25. van Waas M, Neggers SJCMM, Pieters R, Heuvel-Eibrink MM. Components of the metabolic syndrome in 500 adult longterm survivors of childhood cancer. Ann Oncol. 2009;21:1121$-1126$.

26. Pietilä S, Mäkipernaa $\mathrm{A}$, Sievänen $\mathrm{H}$ et al. Obesity and metabolic changes are common in young childhood brain tumor survivors. Pediatr Blood Cancer. 2009;52:853-859.

27. Siviero-Miachon AA, Spinola-Castro AM, Guerra-Junior G. Detection of metabolic syndrome features among childhood cancer survivors: a target to prevent disease. Vasc Health Risk Manag. 2008;4:825-836.

28. Hoffman KE, Derdak J, Bernstein D et al. Metabolic syndrome traits in long-term survivors of pediatric sarcoma. $\mathrm{Pe}$ diatr Blood Cancer. 2008;50:341-346.

29. Chow EJ, Pihoker C, Hunt K, Wilkinson K, Friedman Dl. Obesity and hypertension among children after treatment for acute lymphoblastic leukemia. Cancer. 2007;15:2313-2320.

Submitted: 21 October, 2011

Accepted after reviews: 22 February, 2012 